\title{
Tunable Nb Superconducting Resonator Based on a Constriction Nano-SQUID Fabricated with a Ne Focused Ion Beam
}

\author{
O.W. Kennedy, ${ }^{1,}{ }^{*}$ J. Burnett, ${ }^{1,2}$ J.C. Fenton, ${ }^{1}$ N.G.N. Constantino, ${ }^{1}$ P.A. Warburton, ${ }^{1,3}$ J.J.L. Morton, ${ }^{1,3}$ \\ and E. Dupont-Ferrier ${ }^{1,4, \dagger}$ \\ ${ }^{1}$ London Centre for Nanotechnology, UCL, 17-19 Gordon Street, London, WC1H 0AH, United Kingdom \\ ${ }^{2}$ Microtechnology and Nanoscience, Chalmers University of Technology, SE-41296 Gothenburg, Sweden \\ ${ }^{3}$ Department of Electronic and Electrical Engineering, UCL, London, WC1E 7JE, United Kingdom \\ ${ }^{4}$ Département de Physique, Institut Quantique, Université de Sherbrooke, 2500 Boulevard de l'Université, \\ Sherbrooke, Quebec J1K 2R1, Canada
}

(Received 6 July 2018; revised manuscript received 2 October 2018; published 4 January 2019)

\begin{abstract}
Hybrid superconducting-spin systems offer the potential to combine highly coherent atomic quantum systems with the scalability of superconducting circuits. To fully exploit this potential requires a highquality-factor microwave resonator, tunable in frequency and able to operate at magnetic fields optimal for the spin system. Such magnetic fields typically rule out conventional Al-based Josephson-junction devices that have previously been used for tunable high- $Q$ microwave resonators. The larger critical field of $\mathrm{Nb}$ allows microwave resonators with large field resilience to be fabricated. Here we demonstrate how constriction-type weak links, patterned in parallel into the central conductor of a $\mathrm{Nb}$ coplanar resonator with a neon focused ion beam, can be used to implement a frequency-tunable resonator. We study transmission through two such devices and show how they realize high-quality-factor, tunable, field-resilient devices that hold promise for future applications coupling to spin systems.
\end{abstract}

DOI: 10.1103/PhysRevApplied.11.014006

\section{INTRODUCTION}

A large and growing variety of spin systems have been coupled to superconducting resonators, including ensembles of noninteracting spins in silicon [1], diamond [2], and other materials [3], magnons in ferrimagnets [4] and chiral magnetic insulators [5], and individual spins in quantum dot devices [6]. The motivations for such studies include long-range coupling of spin qubits [7], realization and study of topological systems [8], long-lived microwave quantum memories for superconducting qubits $[9,10]$, and the demonstration of microwave-to-optical conversion at the single-photon level [11]. Planar superconducting circuits provide a robust, well-studied [12], and scalable [13] architecture for such hybrid systems, and superconducting resonators with $Q$ factors greater than $1 \times 10^{6}$ have been achieved [14]. However, for most of the applications described above, externally applied magnetic fields

*oscar.kenedy@ucl.ac.uk

†Eva.Dupont-Ferrier@USherbrooke.ca

Published by the American Physical Society under the terms of the Creative Commons Attribution 4.0 International license. Further distribution of this work must maintain attribution to the author(s) and the published article's title, journal citation, and DOI. from approximately $10 \mathrm{mT}$ to several hundred millitesla, or more, are required to bring the spin systems into a suitable regime of interest. Furthermore, control of the spinresonator coupling is required, often on short timescales, in applications such as quantum memories, and may be achieved, for example, by frequency tuning of the resonator.

Superconducting quantum interference devices (SQUIDs) act as flux-tunable inductors and have been successfully incorporated into resonators to provide frequency tunability [15-17]. The SQUID inductance is tuned from its minimum to its maximum by a change of half a flux quantum in the flux threading the SQUID loop, thus altering the resonator frequency. This means that small local fields provided by on-chip flux lines are able to tune SQUID-embedded resonators on timescales of a few nanoseconds [16]. Technologies that use direct currents to tune the kinetic inductance and hence resonant frequency of resonators have also been developed $[18,19]$ and coupled to spin ensembles [2]. Previous SQUID-tunable devices were fabricated from aluminum with shadowevaporated junctions [16] or used $\mathrm{Nb} / \mathrm{Al}-\mathrm{AlO}_{x} / \mathrm{Nb}$ trilayer junctions [15]. Al devices may suffer from the low critical field of $\mathrm{Al}$ and are expected to have poor magnetic field resilience. $\mathrm{AlO}_{x}$ tunnel junctions may introduce extra losses to resonators and limit quality factors. An 


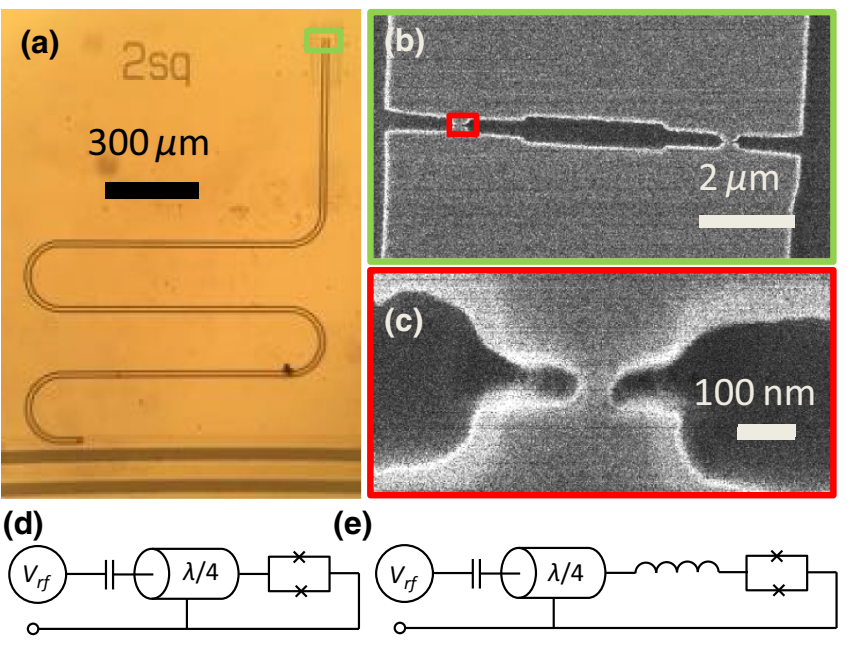

FIG. 1. (a) Optical micrograph of resonator ATune (see the text) with the microwave feed line shown at the bottom. The green rectangle indicates the location of (b), a He-FIB micrograph, obtained with use of an electron flood gun, showing the SQUID loop at the grounded end of ATune. The red rectangle indicates the location of (c), a Ne-FIB-milled constriction with a width of $50 \mathrm{~nm}$. In (a)-(c), the lighter tones show the $\mathrm{Nb}$ and the darker tones show the Si. (d),(e) Equivalent-circuit diagrams of the tunable resonators for (d) ATune and (e) BTune. The lumped-element inductor is present in BTune and not in ATune.

alternative technology is based on nano-SQUIDs [20,21] formed by superconducting-nanowire-based constriction junctions; these have already been shown to possess exceptional field resilience [22].

Nano-SQUIDs are commonly fabricated [23] by a Gabased focused ion beam (FIB); however, this technique has been shown to induce loss into superconducting resonators [24]. Here we use a neon FIB (a technique shown to be compatible with high-quality superconducting resonators [25]) to create constrictions in the central conductor of a $\mathrm{Nb}$ superconducting $\lambda / 4$ coplanar resonator. These constrictions have a width of $50 \mathrm{~nm}$ and are placed in parallel such that they complete a superconducting loop between the current antinode of the resonator and the ground (Fig. 1). We study the microwave transmission of two such devices fabricated from $\mathrm{Nb}$ films of different quality as determined by measuring the quality of bare resonators fabricated from the same films. One such device includes a lumped-element inductor that reduces the tunability of the resonator but may also be used to couple more strongly to spins in future experiments. We demonstrate that nano-SQUID-embedded resonators may realize high-quality, frequency-tunable, and field-resilient resonators.

\section{EXPERIMENTAL}

Coplanar resonators are fabricated by etching thin films of superconductor using a method similar to that described in Ref. [25]. Here $\mathrm{Nb}$ is used, instead of $\mathrm{NbN}$, because of its longer coherence length of $38 \mathrm{~nm}$ [26], which sets the length scale for the width of constrictions needed to make junctions. $\mathrm{Nb}$ thus allows approximately-50-nm constrictions to be used, and these are easier to achieve than the approximately-20-nm constrictions required in $\mathrm{NbN}$. $\mathrm{Nb}$ also has a lower kinetic inductance (hence lower impedance and larger zero-point current fluctuations), which could enable stronger magnetic coupling of the $\mathrm{Nb}$ resonator to spins. $\mathrm{Nb}$ films are deposited on $\mathrm{Si}$ substrates by dc magnetron sputtering from a $99.99 \%$-pure elemental $\mathrm{Nb}$ target in argon. The pressure before deposition is $6 \times 10^{-7}$ mbar and during deposition is $3.5 \times 10^{-3}$ mbar. The sputter power is $200 \mathrm{~W}$, with the deposition timed to produce a 50-nm-thick film.

Two chips are fabricated from $\mathrm{Nb}$ films deposited according to the same recipe in separate sputter runs and labeled "A" and "B." We discuss two resonators from each of these chips, one tunable and one bare resonator labeled "Tune" and "Bare," respectively. We refer to resonators by a combination of chip and resonator label; for example, ATune is the tunable resonator from chip A. Chip A (chip B) has an area of $58 \mathrm{~mm}^{2}\left(44 \mathrm{~mm}^{2}\right)$. Before deposition, chip B is dipped into HF for approximately $10 \mathrm{~s}$ to remove a native oxide coating and then loaded into the sputter system and put under a vacuum approximately 5 min. Quarter-wave $(\lambda / 4)$ resonators [see Fig. 1(a)] with an embedded superconducting loop at the grounded end of the resonator are patterned by electron-beam lithography (EBL) [Fig. 1(b)] in the same lithography step as a microwave feed line. The resist pattern is transferred into the film by a reactive-ion-etch process using a 2:1 ratio of $\mathrm{SF}_{6}$ to $\mathrm{Ar}$ at $30 \mathrm{mbar}$ and $30 \mathrm{~W}$ for $120 \mathrm{~s}$. The reactiveion-etch process additionally etches exposed $\mathrm{Si}$ to a depth of $500 \mathrm{~nm}$, leaving areas with $\mathrm{Nb}$ raised above their surroundings. Resonators on chip B have a long constriction $\left(100 \times 1 \mu \mathrm{m}^{2}\right)$ embedded into the central conductor just before the SQUID. The narrow constriction creates larger local fluctuating fields and would increase the resonator coupling to spins in future devices [1]. The increased coupling comes at the cost of including a constriction that acts as a lumped-element inductor reducing the inductance ratio between the SQUID and the resonator, which consequently reduces the resonator tunability.

The superconducting loop has two constrictions [see Figs. 1(b) and 1(c)]: broad constrictions are defined in the initial EBL exposure and subsequently narrowed to approximately $50 \mathrm{~nm}$ by Ne FIB milling, in which a beam of Ne ions, accelerated to $15 \mathrm{kV}$, mills through the $\mathrm{Nb}$. A dose of approximately $2 \mathrm{nC} / \mu \mathrm{m}^{2}$ is used. Circuit diagrams of these devices are shown in Figs. 1(d) and 1(e) for ATune and BTune, respectively. On chip A, 21 of 22 constrictions milled are still intact after narrowing to a dimension approaching the coherence length, suggesting a high yield for this part of the processing; 
see Supplemental Material [27] for statistical analysis on junction widths.

Resonators are measured at temperature $T \approx 300 \mathrm{mK}$ in a ${ }^{3} \mathrm{He}$ cryostat with a heavily attenuated microwave in line and a cryogenic high-electron-mobility-transistor amplifier on the microwave out line. The $S_{21}$ transmission of microwaves through the feed line - to which the resonator is capacitively coupled - is measured with a Rohde \& Schwarz ZNB8 vector network analyzer. Perpendicular magnetic fields are applied by a superconducting magnet connected to a precision current source (Keithley 2400 SourceMeter). Samples are enclosed in a brass box lined with Eccosorb CR-117, a microwave absorber, to reduce the number of quasiparticles excited by stray IR photons, which we previously showed can have a significant effect on superconducting constrictions [25].

\section{RESULTS}

We first characterize the zero-field characteristics of the resonators on the two chips at the base temperature of the cryostat. In Fig. 2, we show the zero-field magnitude response of $S_{21}$ from ATune [Fig. 2(a)] and BTune [Fig. 2(c)] at an applied power of $-106 \mathrm{dBm}$. Using a traceable fit routine [28], based on fitting a circle to the resonance in the real-imaginary plane, we extract the resonator parameters (fits shown in red in Fig. 2). At an applied power of approximately $-106 \mathrm{dBm}$, ATune, ABare, BTune, and BBare have internal quality factors $Q_{i}=2.8 \times 10^{4}, 5.3 \times 10^{4}, 1.25 \times 10^{5}$, and $1.26 \times 10^{5}$, respectively (resonance notches of bare resonators are not shown). The biggest difference between zero-field quality factors is between the different films rather than between the bare and tunable resonators. This shows that, at high drive power, the SQUIDs introduce minimal extra losses and that the losses are dominated by effects arising from the film quality. The asymmetry of the resonance of ATune [Figs. 2(a) and 2(b)] persists down to single-photon powers due to an impedance mismatch, and this is fully captured by the fit routine. Figures 2(b) and 2(d) shows that both ATune and BTune resonances are present at applied perpendicular fields approaching $0.5 \mathrm{mT}$ with resonance responses. We discuss resonator behavior in an applied magnetic field later.

We measure the internal losses of all four resonators $\left(\delta_{i}=1 / Q_{i}\right)$ as a function of the average photon number within the resonator [see Fig. 2(e)]. Resonators from film A show higher losses across all powers than resonators from film B, irrespective of whether they are bare or tunable. While ATune shows more losses than $\mathrm{ABare}$ and a greater increase in losses at low power, for film B the losses are approximately equal for BTune and BBare. We therefore attribute the difference in losses in film A to on-chip variation in film quality, as commonly
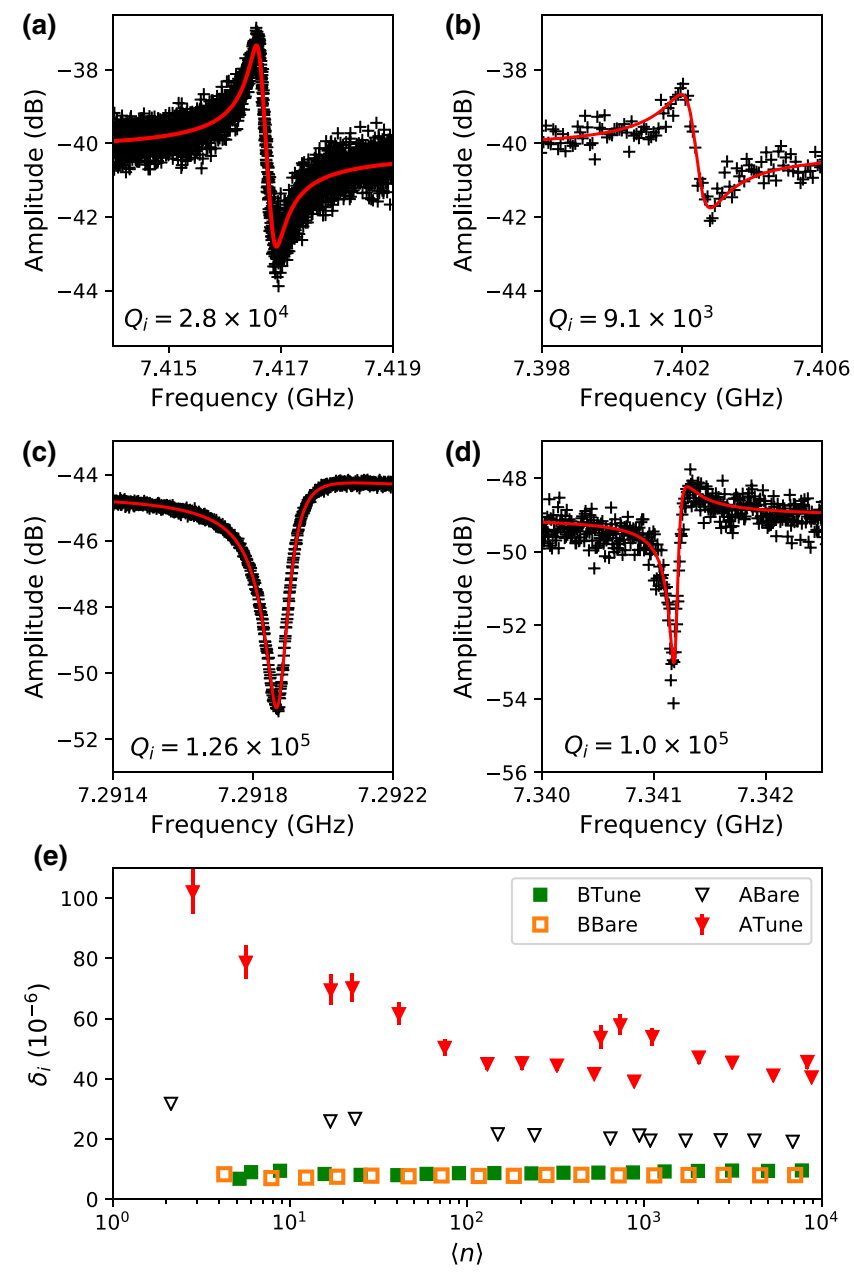

FIG. 2. $S_{21}$ amplitude response (black crosses) and fits (red line) for (a),(b) ATune and (c),(d) BTune. Measurements are performed at (a),(c) zero field and (b),(d) approximately $0.5 \mathrm{mT}$ applied perpendicular field. All responses are recorded at a power of $-106 \mathrm{dBm}$, and internal quality factors are indicated. The lower frequency of BTune in (c) than in (d) is due to device aging between measurements. The local fields in (b),(d) are approximately $60 \mathrm{mT}$ and approximately $20 \mathrm{mT}$, respectively. (e) Internal losses of all four resonators as a function of average photon number stored in the resonator. Error bars are shown where they are larger than the markers.

seen in coplanar-waveguide resonators. Losses are dominated by losses associated with the film rather than losses introduced by the SQUID even in the single-photon limit. The difference between films could be a result of the small recipe differences, such as the HF dip for film B; however, the increased loss at high powers is indicative of increased conductor losses associated with the quality of superconducting films.

\section{A. Tunability}

To study resonator tuning we examine ATune. Without a lumped-element inductor, the tunable SQUID inductance 
is a greater fraction of the total inductance, and so this resonator tunes more in frequency. We first study its behavior in a small perpendicular magnetic field $(10 \mu \mathrm{T}$ or less). Fig. 3(a) shows a typical field sweep; this reveals the smooth tuning of the resonator toward lower frequencies as the magnetic field amplitude is increased. The full range of frequency tuning is found to be approximately 100 $\mathrm{MHz}$, obtained by changing the applied perpendicular field by approximately $10 \mu \mathrm{T}$ [see Fig. 3(b)] [29]. To analyze this behavior, we start by assuming a Josephson-like sinusoidal current-phase relationship for the nanoconstrictions since the length:width ratio of the constrictions shown in Fig. 1(c) is approximately 1 [30]. We therefore treat the superconducting loop as a dc SQUID with a flux-tunable inductance [16]:

$$
L_{\text {SQUID }}=\Phi_{0} /\left(4 \pi I_{C 0}|\cos f|\right),
$$

where $I_{\mathrm{C} 0}$ is the zero-field critical current of the SQUID, $f=\pi \Phi / \Phi_{0}$ is the frustration of the SQUID when threaded by flux $\Phi$, and $\Phi_{0}$ is the flux quantum.

The total impedance $Z_{T}$ of a transmission line terminated by a SQUID at frequency $v$ is given by

$$
Z_{T}=\frac{Z\left[Z_{\mathrm{SQUID}}+j Z \tan (2 \pi v d / v)\right]}{\left[Z+j Z_{\mathrm{SQUID}} \tan (2 \pi v d / v)\right]},
$$

where $d$ is the length of the transmission line, $Z$ is the impedance of the transmission line, $Z_{\text {SQUID }}$ is the impedance of the SQUID, and $v=\sqrt{1 / l_{0} c_{0}}$ is the speed of light in the transmission line, where $c_{0}\left(l_{0}\right)$ is the capacitance (inductance) per unit length. $Z_{T}$ is real at resonance, and the resonant frequencies $v_{i}$ are therefore given by

$$
\tan \left(\frac{2 \pi v_{i} d}{v}\right)=\frac{\left|Z_{T}\right|}{l_{0} v}
$$

which may be solved numerically. The fundamental resonant frequency may be expressed approximately [31] in terms of the total inductance $L$ and capacitance $C$ of the distributed resonator:

$$
\nu_{0}(B)=\frac{1}{4 \sqrt{\left[L_{\mathrm{res}}+L_{\mathrm{SQUID}}^{0}+\Delta L(B)\right] C}},
$$

where $L_{\text {res }}$ is the inductance of the resonator excluding the SQUID, $L_{\text {SQUID }}^{0}$ is the zero-field inductance of the SQUID, and $\Delta L(B)$ is the change of inductance of the SQUID with the field, which, from Eq. (1), is equal to $\Phi_{0} / 4 \pi I_{\mathrm{C} 0} \times$ $(1 /|\cos f|-1)$. Assuming $f \propto B$, an assumption which we examine further below, we can write

$$
\frac{\nu_{0}(B)}{\nu_{0}(0)}=\sqrt{\frac{1}{1+\frac{\Delta L(B)}{L_{\mathrm{res}}+L_{\mathrm{SQUID}}^{0}}}}=\sqrt{\frac{1}{1-A+\frac{A}{|\cos (K B)|}}},
$$

where $A=\Phi_{0} /\left[4 \pi I_{\mathrm{C} 0}\left(L_{\text {res }}+L_{\text {SQUID }}^{0}\right)\right]$ and $f=K B$, so $K$ scales the field to $f$. The observed $v_{0}(B)$ dependence of the resonator in Fig. 3 fits well with Eq. (5), allowing determination of $A$ and $K$.

We next consider the relation between the field periodicity of the tuning behavior and the flux quantum. SQUID behavior is periodic in applied flux. The area of the SQUID loop in ATune is $A_{\text {loop }}=3.7 \pm 0.3 \mu \mathrm{m}^{2}$, so $10 \mu \mathrm{T}$ (the

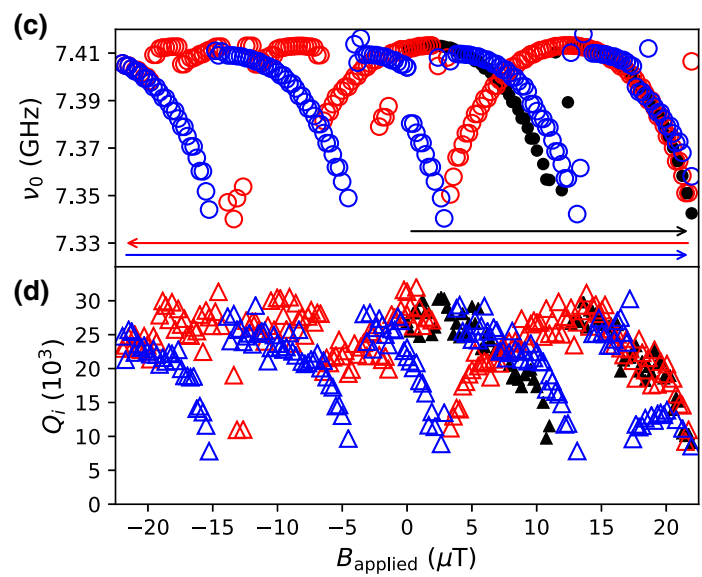

FIG. 3. (a) The tuning of resonant frequency (circles) and quality factor (triangles) of ATune as the magnetic field is swept. The resonant frequency is fitted by Eq. (5), with fitting parameters $A=0.050$ and $K=0.101 \mu \mathrm{T}^{-1}$. For the data shown, the central value $\Delta B=0$ corresponds to an applied field $B=12.4 \mu \mathrm{T}$. (b) Tuning of the resonator when the field is changed in $0.1-\mu \mathrm{T}$ steps from $B=12.3 \mu \mathrm{T}$. A maximal detuning of the resonator of $-101 \mathrm{MHz}$ is obtained. (c) Resonant frequency and (d) internal quality factor of ATune as the applied field is swept from 0 to $22 \mu \mathrm{T}$ (black), then down from $22 \mu \mathrm{T}$ to $-22 \mu \mathrm{T}$ (red) and then back up from $-22 \mu \mathrm{T}$ to $22 \mu \mathrm{T}$ (blue). The sweep directions are shown by arrows at the bottom of (c). 
field required to maximally tune the resonator) corresponds to a flux $B A_{\text {loop }} \approx 0.02 \Phi_{0}$. Assuming the tuning arises from the SQUID, the field required to maximally tune the resonator implies that the local flux density at the SQUID is much greater than the $10 \mu \mathrm{T}$ applied by the magnet. This indicates substantial flux focusing due to flux expulsion from the superconducting ground plane surrounding the resonators. We return to the topic of flux focusing later.

As ATune is tuned away from $v_{0}, Q_{i}$ drops from its maximum value, $2.8 \times 10^{4}$, to $1.0 \times 10^{4}$ when maximally tuned. The same trend of $Q_{i}$ decreasing with tuning is seen in BTune. This phenomenon of $Q_{i}$ decreasing with tuning was previously observed and attributed to increasing thermal noise as the SQUID is tuned [15] or increased dissipation caused by a subgap resistance [16]. An alternative explanation could be that dilute surface spins [32] induce spectral broadening of the resonance line shape by flux-noise-based frequency jitter in these flux-tunable resonators. However, even for the highest values of flux noise in Ref. [33], which correspond to approximately $100 \mu \Phi_{0}$, the corresponding frequency jitter would be too small to create sufficient spectral broadening to explain the drop in $Q_{i}$ observed here. The source of these extra losses is the subject of ongoing work.

\section{B. Hysteresis and premature switching}

When ATune is tuned over more than one period, as shown in Figs. 3(c) and 3(d), a hysteretic behavior is seen, similar to that previously reported in Al constriction SQUID resonators [17]. In addition, frequency jumps are observed at values of tuning less than the maximum value (see, e.g., the region between -5 and $-20 \mu \mathrm{T}$ ). We attribute jumps at nonmaximal tuning to flux trapping as the field is ramped up and down. The oscillations in the internal quality factor [Fig. 3(d)] follow the frequency tuning of the resonator, showing that the degradation in $Q_{i}$ with magnetic field arises from the state of the SQUID and not from the properties of the resonator in a magnetic field.

The hysteretic tuning of the resonant frequency and internal quality factor may be explained by significant self-inductance of the superconducting SQUID loop. The SQUID has a characteristic parameter $\beta_{L}=2 L_{\text {loop }} I_{C} / \Phi_{0}$ (where $L_{\text {loop }}$ is the inductance of the loop and $I_{\mathrm{C}}$ is the Josephson critical current). When $\beta_{L} \gtrsim 1$, the SQUID behavior becomes hysteretic with applied flux, as shown in Fig. 4(a). The red path in Fig. 4(b) maps out the flux within the SQUID as the field is increased (assuming zero temperature, in the absence of fluctuations). At extremal points, the flux threading the SQUID exhibits discontinuous jumps as $\Phi_{\text {app }}$ is ramped upward, occurring periodically with a period of $2 \Phi_{0}$. At finite temperature, thermal fluctuations cause these jumps to occur at a temperature-dependent flux less than that at $T=0$.

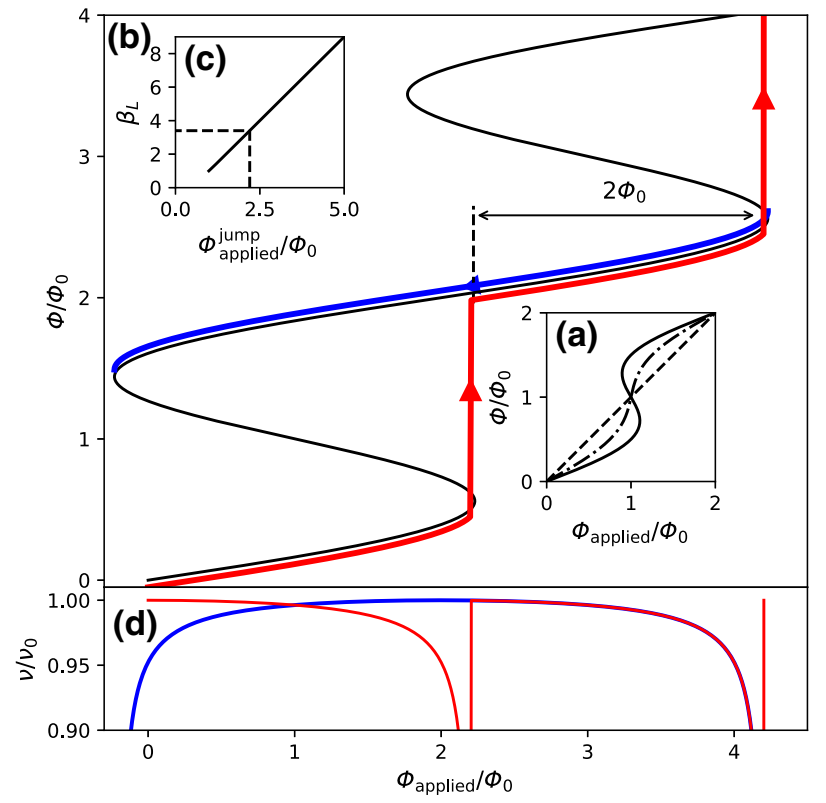

FIG. 4. (a) Variation with applied external flux $\Phi_{\text {applied }}$ of flux $\Phi$ threading the SQUID for SQUIDs with $\beta_{L}=0$ (dashed line), 0.5 (dot-dashed line), and 1 (solid line). (b) Variation with applied flux of flux threading a SQUID with $\beta_{L}=3.4$. The black line shows the solution to the equation governing flux threading a hysteric SQUID [34]. The red (blue) line indicates how the flux threading the SQUID evolves in the absence of fluctuations with the applied flux ramping upward (downward) as in Figs. 3(c) and 3(d). (c) The relationship between applied flux required to maximally detune the resonator and $\beta_{L}$ of the embedded SQUID. (d) Variation of resonant frequency of the SQUID with applied flux depending on how the field is ramped, calculated with an inductance ratio between the SQUID and the resonator determined from the parameter $A$ from the fit in Fig. 3(a). The colors of the lines correspond to the same flux ramping as in (b).

Using this $2 \Phi_{0}$ periodicity, we are able to calibrate local fields at our device and experimentally quantify flux focusing in these hysteretic devices. Jumps occur every $9.7 \mu \mathrm{T}$ (averaged over four consecutive jumps in Fig. 5), which corresponds to $B A_{\text {loop }} \approx 0.016 \Phi_{0}$. Identifying the jumps as $2 \Phi_{0}$-periodic features, we infer a flux focusing $\mathcal{F} \approx 124$, where we define $\mathcal{F}$ by $B_{\text {local }}=\mathcal{F} B$. Significant flux focusing from superconducting ground planes was recently investigated theoretically and experimentally in Ref. [35], where simulations gave $\mathcal{F} \approx 27.5$. The extent of flux focusing is specific to the device geometry; for example, features designed to trap flux can have large effects on $\mathcal{F}$.

The change in applied flux required to maximally tune the resonator frequency is determined by the $\beta_{L}$ value of the SQUID [see Fig. 4(c)]. At finite temperature, the flux in the SQUID jumps before reaching the point of instability shown in Fig. 4, and so the experimentally measured jump positions provide a lower bound on $\beta_{L}$. We thus infer that $\beta_{L}>3.4$ for our device. Using Eq. (1) and the fit 
parameter $A$ (which relates the inductance of the resonator and the inductance of the SQUID), we can calculate the expected tuning of the resonator in an applied magnetic field on the basis of a sinusoidal current-phase relation [Fig. 4(d)]. The smooth tuning shown in Fig. 3(a) [blue line in Figs. 4(b) and 4(d)] and the jumps seen in Fig. 3(c) [red line in Figs. 4(b) and 4(d)] are qualitatively reproduced. The calculation, however, suggests that resonant frequencies should decrease significantly in the vicinity of hysteretic jumps due to the asymptote in $1 / \cos f$ at $f=1 / 2$, whereas in practice these resonators tune by only approximately $1 \%$ of their untuned frequency. Numerical analysis [36] based on Eq. (3) predicts such reduced tuning for resonators where the SQUID inductance or capacitance is a significant fraction of the total inductance or capacitance of the distributed resonator. We determine the untuned inductance of the SQUID to be approximately $10 \%$ of the total inductance of the distributed resonator (see Supplemental Material [27] for details), which in Ref. [36] is sufficient (even with small SQUID capacitance) to reduce the tuning of the resonator approaching $\Phi=\Phi_{0} / 2$ to around only $30 \%$. Additionally, asymmetry between junctions allows switching at smaller tuning than for perfectly symmetric devices as the narrower junction becomes maximally biased (and hence switches) before the wider junction becomes maximally biased.

\section{Magnetic field resilience}

In Fig. 5, ATune (black symbols), ABare (red symbols), and BTune (green circles and magenta triangles) are measured as the applied perpendicular magnetic field is increased from zero to approximately $0.5 \mathrm{mT}$, which corresponds to a local field at the SQUID of approximately 60 $\mathrm{mT}$ for chip A and approximately $20 \mathrm{mT}$ for chip B due to different flux-focusing factors. The smaller size of chip B likely contributes to the smaller flux-focusing factor.
The resonance responses and their fits at these fields are shown in Figs. 2(b) and 2(c). Even at these perpendicular magnetic fields, the internal quality factor $Q_{i}$ of ATune (BTune) is $9.1 \times 10^{3}\left(9.98 \times 10^{4}\right)$ and the resonator remains tunable, demonstrating the field resilience of these devices. Figure 5(c) shows that BTune retains a high quality factor across the whole field range shown, even after tuning through 42 periods.

For ABare, $v_{0}$ and $Q_{i}$ tune weakly and smoothly as the applied magnetic field is increased to about $0.21 \mathrm{mT}$, with the exception of abrupt drops in both $v_{0}$ and $Q_{i}$ at four field values up to $0.2 \mathrm{mT}$ (see Fig. 5). This steplike response of $Q_{i}$ versus magnetic field is consistent with the formation of vortices on the superconducting resonator's central conductor [37]. For ATune, $Q_{i}$ changes by a factor of about 5 with the field as the resonator tunes (as shown in Fig. 3). In addition to the modulation with tuning, the maximum $Q_{i}$ also drops with increasing applied field. The magnitude and field scale of this drop are similar to those of the bare resonator. The respective $Q_{i}(B)$ dependences for ATune and ABare are consistent with the maximum of the field-modulated $Q_{i}$ in ATune being limited, as $B$ increases, by the same physics that causes the steplike reductions in $Q_{i}$ for ABare. The similarity is seen even more clearly in $v_{0}(B)$, where the untuned resonant frequency of ATune jumps at an applied field of $0.21 \mathrm{mT}$ just as in the bare resonator. This suggests that not only does the nano-SQUID introduce minimal loss at zero applied field but also that the resonator's field resilience is the limiting factor in the field performance of the device. This is also consistent with measurements of nano-SQUIDs fabricated from ultrathin niobium films successfully operating in parallel fields up to $7 \mathrm{~T}$ [38]. Our results are therefore promising for future generations of devices where SQUIDs are embedded within field-resilient resonators. Importantly, ATune operates at local fields comfortably above $30 \mathrm{mT}$ (and BTune looks likely also to
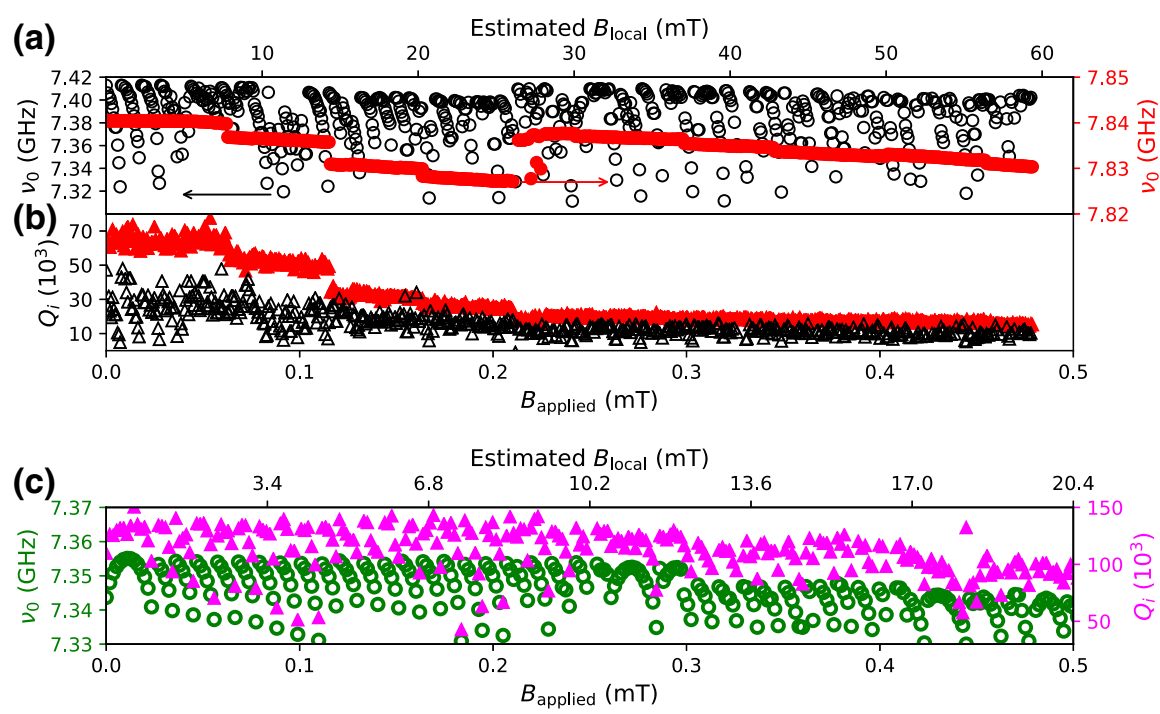

FIG. 5. Magnetic field dependence of (a) resonant frequency and (b) internal quality factor for ATune (black symbols) and ABare (red symbols). The local field $B_{\text {local }}$ is estimated on the basis of measurements of flux focusing (see the main text). (c) Magnetic field dependence of resonant frequency (green circles) and internal quality factor (magenta triangles) for BTune. The local field is determined as for ATune. 
operate at such fields), the first clock transition of bismuth spins.

In the literature, resonator tuning is typically given in units of flux quanta. This means that there are no comparable results on field resilience of tunable resonators to which this device can be compared. Therefore we believe that this study is the first report addressing the field resilience of tunable resonators.

\section{CONCLUSIONS}

In conclusion, we have embedded constriction nanoSQUIDs in $\mathrm{Nb}$ resonators and show that this device geometry results in a frequency-tunable resonator, with $100 \mathrm{MHz}$ of tuning demonstrated in a $7.42-\mathrm{GHz}$ resonator. We fabricate these devices from two $\mathrm{Nb}$ chips and show that the untuned quality factor of these resonators is limited by the bare-resonator technology rather than embedding the SQUID, up to an internal quality factor $Q_{i}$ of $1.2 \times 10^{5}$-the highest quality factor of a SQUID-tunable resonator reported to date. These results are realized in two different resonators, where one has high quality and the other is more tunable; however, there is no fundamental trade-off being between quality and tunability in these devices. By comparing the quality factors of tunable and bare resonators, we demonstrate that the quality is determined by the superconducting films. The tunability differs due to different device geometries. We show that even these high quality factors are resonatorlimited both at zero field and at applied perpendicular magnetic fields up to $0.5 \mathrm{mT}$ and for resonator photon number from $10^{4}$ down to single photons at zero field. These resonators remain tunable at applied perpendicular fields of $0.5 \mathrm{mT}$ and retain an untuned quality factor of approximately $1 \times 10^{5}$. The devices presented here compare favorably with the highest-quality tunable resonators previously reported [39]. Those resonators tune due to kinetic inductance shifts from direct currents and achieve a high-power quality factor of $1.8 \times 10^{5}$; there are no reports of low-power operation.

A number of modifications to the device and measurement setup may straightforwardly be made to improve field resilience: specifically, operating the device in parallel fields (which tune spins to the clock transition without the application of large fields to the resonator), modifying the device design by the addition of antidots [40], patterning the whole ground plane [41], and/or the use of resonator designs inherently more robust to an external field [40-42]. The nano-SQUIDs allow us to measure the local magnetic field around the resonators, and we find that the local fields are 30-120 times greater than the applied fields, meaning these modifications should straightforwardly significantly improve field resilience.

These resonators hold great promise for future hybrid quantum system applications. For example, a tunable resonator operating at $27 \mathrm{mT}$ coupled to $\mathrm{Bi}$ spins in $\mathrm{Si}$ could address spins at their clock transition. The resonator could be tuned into resonance with the spins, and ESR pulse sequences could be applied, before the resonator is tuned away from the spins in frequency, allowing the long-coherence-time spins to store quantum information, unperturbed by the resonators.

\section{ACKNOWLEDGMENTS}

The authors thank E.J. Romans, A. Blois, S. Probst, P. Bertet, and C.W. Zollitsch for useful discussions. This project received funding from the European Union's Horizon 2020 research and innovation program under Marie Sklodowska-Curie Grant Agreement No. 705713 QUINTESSENS (E.D.-F.), the European Union's Seventh Framework Programme through Grant Agreement No. 279781 (ASCENT) (J.M.), Carl Zeiss Semiconductor Manufacturing (O.W.K.), and the UK Engineering and Physical Sciences Research Council, Grants No. EP/J017329/1 (J.C.F.), No. EP/K024701/1 and No. EP/H005544/1 (O.W.K. and P.A.W.), and No. EP/P510270/1 (O.W.K).

[1] A. Bienfait, J. J. Pla, Y. Kubo, X. Zhou, M. Stern, C. C. Lo, C. D. Weis, T. Schenkel, D. Vion, D. Esteve, J. J. L. Morton, and P. Bertet, Controlling spin relaxation with a cavity, Nature 531, 74 (2016).

[2] Y. Kubo, F. R. Ong, Patrice Bertet, Denis Vion, V. Jacques, D. Zheng, A. Dréau, J.-F. Roch, Alexia Auffèves, Fedor Jelezko, J. Wrachtrup, M. F. Barthe, P. Bergonzo, and D. Esteve, Strong Coupling of a Spin Ensemble to a Superconducting Resonator, Phys. Rev. Lett. 105, 140502 (2010).

[3] S. Probst, H. Rotzinger, S. Wünsch, P. Jung, M. Jerger, M. Siegel, A. V. Ustinov, and P. A. Bushev, Anisotropic Rare-Earth Spin Ensemble Strongly Coupled to a Superconducting Resonator, Phys. Rev. Lett. 110, 157001 (2013).

[4] H. Huebl, C. W. Zollitsch, J. Lotze, F. Hocke, M. Greifenstein, A. Marx, R. Gross, and S. T. B. Goennenwein, High Cooperativity in Coupled Microwave Resonator Ferrimagnetic Insulator Hybrids, Phys. Rev. Lett. 111, 127003 (2013).

[5] L. V. Abdurakhimov, S. Khan, N. A. Panjwani, J. D. Breeze, S. Seki, Y. Tokura, J. J. L. Morton, and H. Kurebayashi, Strong coupling between magnons in a chiral magnetic insulator $\mathrm{Cu}_{2} \mathrm{OSeO}_{3}$ and microwave cavity photons, arXiv:1802.07113 (2018).

[6] Xiao Mi, J. V. Cady, D. M. Zajac, P. W. Deelman, and J. R. Petta, Strong coupling of a single electron in silicon to a microwave photon, Science 355, 156 (2017).

[7] C. Grèzes, Y. Kubo, B. Julsgaard, T. Umeda, J. Isoya, H. Sumiya, H. Abe, S. Onoda, T. Ohshima, K. Nakamura, I. Diniz, A. Auffeves, V. Jacques, J.-F. Roch, D. Vion, D. Esteve, K. Moelmer, and P. Bertet, Towards a spinensemble quantum memory for superconducting qubits, $\mathrm{C}$. R. Phys. 17, 693 (2016). 
[8] T. W. Larsen, K. D. Petersson, F. Kuemmeth, T. S. Jespersen, P. Krogstrup, J. Nygrd, and C. M. Marcus, Semiconductor-nanowire-based superconducting qubit, Phyis. Rev. Lett. 115, 127001 (2015).

[9] F. Yan, S. Gustavsson, A. Kamal, J. Birenbaum, A. P. Sears, D. Hover, T. J. Gudmundsen, D. Rosenberg, G. Samach, S. Weber, J. L. Yoder, T. P. Orlando, J. Clarke, A. J. Kerman, and W. D. Oliver, The flux qubit revisited to enhance coherence and reproducibility, Nat. Commun. 7, 12964 (2016).

[10] A. Dunsworth, A. Megrant, C. Quintana, Z. Chen, R. Barends, B. Burkett, B. Foxen, Y. Chen, B. Chiaro, A. Fowler, R. Graff, E. Jeffrey, J. Kelly, E. Lucero, J. Y. Mutus, M. Neeley, C. Neill, P. Roushan, D. Sank, A. Vainsencher, J. Wenner, T. C. White, and J. M. Martinis, Characterization and reduction of capacitive loss induced by sub-micron Josephson junction fabrication in superconducting qubits, Appl. Phys. Lett. 111, 022601 (2017).

[11] S. Blum, C. O'Brien, N. Lauk, P. Bushev, M. Fleischhauer, and G. Morigi, Interfacing microwave qubits and optical photons via spin ensembles, Phyis. Rev. A 91, 033834 (2015).

[12] J. Burnett, L. Faoro, I. Wisby, V. L. Gurtovoi, A. V. Chernykh, G. M. Mikhailov, V. A. Tulin, R. Shaikhaidarov, V. Antonov, P. J. Meeson, A. Y. Tzalenchuk, and T. Lindström, Evidence for interacting two-level systems from the $1 /$ f noise of a superconducting resonator, Nat. Commun. $\mathbf{5}$, 4119 (2014).

[13] R. Barends, J. Kelly, A. Megrant, A. Veitia, D. Sank, E. Jeffrey, T. C. White, J. Mutus, A. G. Fowler, B. Campbell, Y. Chen, Z. Chen, B. Chiaro, A. Dunsworth, C. Neill, P. O'Malley, P. Roushan, A. Vainsencher, J. Wenner, A. N. Korotkov, A. N. Cleland, and J. M. Martinis, Superconducting quantum circuits at the surface code threshold for fault tolerance, Nature 508, 500 (2014).

[14] A. Megrant, C. Neill, R. Barends, B. Chiaro, Yu Chen, L. Feigl, J. Kelly, Erik Lucero, Matteo Mariantoni, P. J. J. O’Malley, D. Sank, A. Vainsencher, J. Wenner, T. C. White, Y. Yin, J. Zhao, C. J. Palmstrm, John M. Martinis, and A. N. Cleland, Planar superconducting resonators with internal quality factors above one million, Appl. Phys. Lett. 100, 113510 (2012).

[15] A. Palacios-Laloy, F. Nguyen, F. Mallet, P. Bertet, D. Vion, and D. Esteve, Tunable resonators for quantum circuits, J. Low. Temp. Phys. 151, 1034 (2008).

[16] M. Sandberg, C. M. Wilson, F. Persson, T. Bauch, G. Johansson, V. Shumeiko, T. Duty, and P. Delsing, Tuning the field in a microwave resonator faster than the photon lifetime, Appl. Phys. Lett. 92, 203501 (2008).

[17] E. M. Levenson-Falk, R. Vijay, and I. Siddiqi, Nonlinear microwave response of aluminum weak-link Josephson oscillators, Appl. Phys. Lett. 98, 3115 (2011).

[18] A. A. Adamyan, S. E. Kubatkin, and A. V. Danilov, Tunable superconducting microstrip resonators, Appl. Phys. Lett. 108, 172601 (2016).

[19] A. T. Asfaw, A. J. Sigillito, A. M. Tyryshkin, T. Schenkel, and S. A. Lyon, Multi-frequency spin manipulation using rapidly tunable superconducting coplanar waveguide microresonators, Appl. Phys. Lett. 111, 032601 (2017).
[20] C. Granata, and A. Vettoliere, Nano superconducting quantum interference device: A powerful tool for nanoscale investigations, Phys. Rep. 614, 1 (2016).

[21] M. J. Martńez-Pérez, R. Kleiner, and D. Koelle, in The Oxford Handbook of Small Superconductors (OUP, Oxford, 2017).

[22] T. Schwarz, J. Nagel, R. Wölbing, M. Kemmler, R. Kleiner, and D. Koelle, Low-noise nano superconducting quantum interference device operating in tesla magnetic fields, ACS Nano 7, 844 (2013).

[23] L. Hao, D. C. Cox, and J. C. Gallop, Characteristics of focused ion beam nanoscale Josephson devices, Supercond. Sci. Technol. 22, 064011 (2009).

[24] M. D. Jenkins, U. Naether, M. Ciria, J. Sesé, J. Atkinson, C. Sánchez-Azqueta, E. del Barco, J. Majer, D. Zueco, and F. Luis, Nanoscale constrictions in superconducting coplanar waveguide resonators, Appl. Phys. Lett. 105, 162601 (2014).

[25] J. Burnett, J. Sagar, O. W. Kennedy, P. A. Warburton, and J. C. Fenton, Low-Loss Superconducting Nanowire Circuits using a Neon Focused Ion Beam, Phys. Rev. Appl. 8, 014039 (2017).

[26] R. Meservey, and B. B. Schwartz, Equilibrium properties: Comparison of experimental results with predictions of the BCS theory, type Tech. Rep. (Massachusetts Inst. of Tech., Cambridge, 1969).

[27] See Supplemental Material at http://link.aps.org/supple mental/10.1103/PhysRevApplied.11.014006 for details on further statistics on junction dimensions, measurements on additional devices, and calculations of resonator and SQUID inductance.

[28] S. Probst, F. B. Song, P. A. Bushev, A. V. Ustinov, and M. Weides, Efficient and robust analysis of complex scattering data under noise in microwave resonators, Rev. Sci. Instrum. 86, 024706 (2015).

[29] The field is changed in small steps $(0.1 \mu \mathrm{T})$ to approach maximal detuning while ensuring that the point of maximal detuning is not overshot before the sweep direction is reversed.

[30] A. A. Golubov, M. Y. Kupriyanov, and E. Il'ichev, The current-phase relation in Josephson junctions, Rev. Mod. Phys. 76, 411 (2004).

[31] P. J. Jones, J. A. M. Huhtamäki, J. Salmilehto, K. Y. Tan, and M. Möttönen, Tunable electromagnetic environment for superconducting quantum bits, Sci. Rep. 3, 1987 (2013).

[32] S. E. de Graaf, A. A. Adamyan, T. Lindström, D. Erts, S. E. Kubatkin, A. Ya Tzalenchuk, and A. V. Danilov, Direct Identification of Dilute Surface Spins on $\mathrm{Al}_{2} \mathrm{O}_{3}$ : Origin of Flux Noise in Quantum Circuits, Phyis. Rev. Lett. 118, 057703 (2017).

[33] G. Ithier, E. Collin, P. Joyez, P. J. Meeson, D. Vion, D. Esteve, F. Chiarello, A. Shnirman, Y. Makhlin, J. Schriefl, and G. Schön, Decoherence in a superconducting quantum bit circuit, Phyis. Rev. B 72, 134519 (2005).

[34] M. Tinkham, Introduction to Superconductivity (Courier Corporation, North Chelmsford, 2004).

[35] D. Bothner, D. Wiedmaier, B. Ferdinand, R. Kleiner, and D. Koelle, Improving Superconducting Resonators in Magnetic Fields by Reduced Field Focussing and Engineered Flux Screening, Phys. Rev. Appl. 8, 034025 (2017). 
[36] E.-J. Kim, J. R. Johansson, and F. Nori, Circuit analog of quadratic optomechanics, Phys. Rev. A 91, 033835 (2015).

[37] I. Nsanzineza, and B. L. T. Plourde, Trapping a single vortex and reducing quasiparticles in a superconducting resonator, Phyis. Rev. Lett. 113, 117002 (2014).

[38] L. Chen, W. Wernsdorfer, C. Lampropoulos, George Christou, and Irinel Chiorescu, On-chip SQUID measurements in the presence of high magnetic fields, Nanotechnology 21, 405504 (2010).

[39] M. R. Vissers, J. Hubmayr, M. Sandberg, S. Chaudhuri, C. Bockstiegel, and J. Gao, Frequency-tunable superconducting resonators via nonlinear kinetic inductance, Appl. Phys. Lett. 107, 062601 (2015).
[40] D. Bothner, T. Gaber, M. Kemmler, D. Koelle, and R. Kleiner, Improving the performance of superconducting microwave resonators in magnetic fields, Appl. Phys. Lett. 98, 102504 (2011).

[41] S. E. de Graaf, A. V. Danilov, Astghik Adamyan, Thilo Bauch, and S. E. Kubatkin, Magnetic field resilient superconducting fractal resonators for coupling to free spins, J. Appl. Phys. 112, 123905 (2012).

[42] N. Samkharadze, A. Bruno, P. Scarlino, G. Zheng, D. P. Di-Vincenzo, L. DiCarlo, and L. M. K. Vandersypen, HighKinetic-Inductance Superconducting Nanowire Resonators for Circuit QED in a Magnetic Field, Phys. Rev. Appl. 5, 044004 (2016). 